\title{
Lesiones craneales secundarias a parto asistido con forceps
}

\author{
F. Villarejo; J.M. Belinchón; F. Carceller; A. Gómez-Sierra; A. Pascual; F. Cordobés; C. Pérez-Díaz y B. Rivero
}

Servicios de Neurocirugía del Hospital Niño Jesús y Hospital La Paz. Madrid. España.

\section{Resumen}

Los partos asistidos con forceps o vacuum aumentan la incidencia de lesiones craneoencefálicas fetales, siendo la tendencia actual a realizar cesáreas en partos que se prevén difíciles.

Presentamos una serie de tres casos de lesiones craneales secundarias a parto asistido con forceps, dos casos de fracturas deprimidas y una fractura deprimida con hematoma epidural subyacente. El diagnóstico se realiza con la clínica y técnicas de imagen como TAC o IRM. EI tratamiento es quirúrgico en la mayoría de casos, con elevación de la fractura y evacuación del hematoma.

La forma correcta de aplicar los forceps resulta esencial para prevenir lesiones craneales fetales, especialmente en partos difíciles.

PALABRAS CLAVE: Extracción con forceps. Extracción con vaéuum. Fractura craneal. Fractura deprimida. Hematoma epidural.

\section{Cranial lesions due to forceps delivery}

\section{Summary}

Deliveries with forceps or vacuum-extraction increase the incidence of perinatal craneoencephalic lesions, for which reason cesarean sections are performed more frequently. We report 3 cases of cranial lesions due to forceps deliveries, 2 with depressed skull fractures and 1 with a depressed fracture and an associated epidural hematoma. Diagnosis is made on clinical and radiological founds with CT scan or MRI. Treatment is surgical and consists of elevation of the depressed fracture and evacuation of the hematoma. The correct use of forceps is very important to avoid this kind of lesions in the newborn, especially in cases of difficult delivery.
KEYWORDS: Delivery with vacuum-extraction. Depressed skull fracture. Epidural hematoma. Forceps delivery. Skull fractures.

\section{Introducción}

Durante el parto pueden ocurrir múltiples lesiones del sistema nervioso central y periférico, muchas de las cuales pueden prevenirse ${ }^{10}$. La cabeza fetal es sometida a importantes compresiones contra la columna lumbar, promontorio sacro y sínfisis del pubis al atravesar el canal del parto. Si además se añaden compresiones craneales por instrumentaciones ginecológicas pueden producirse lesiones intra o extracraneales, espinales o de nervios periféricos. La incidencia de este tipo de lesiones ha disminuido drásticamente con la práctica cada vez mayor de cesáreas.

\section{Material y métodos}

Presentamos tres casos de lesiones debidas a extracción fetal con forceps: un hematoma epidural con hundimiento craneal y dos fracturas deprimidas.

\section{Caso 1}

Recien nacido de un día de vida después de gestación de 40 semanas y parto asistido con forceps. Es trasladado a nuestro centro por cefalohematoma parietal izquierdo con fractura deprimida parietal y hematoma epidural subyacente (Fig 1). La exploración neurológica fue normal. Fue intervenido evacuándose el hematoma y elevándose la fractura deprimida, dejando un drenaje epidural 24 horas. Fue dado de alta a los 4 días y tras 2 años de seguimiento está asintomático.

\section{Caso 2}

Niña recién nacida con forceps que presenta una gran 


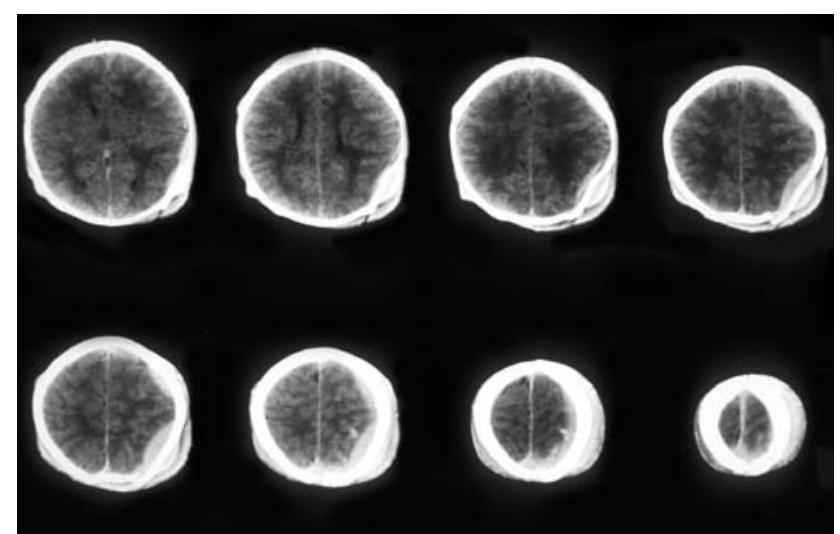

Figura 1. TAC muestra fractura parietal izquierda más hematoma epidural subyacente.

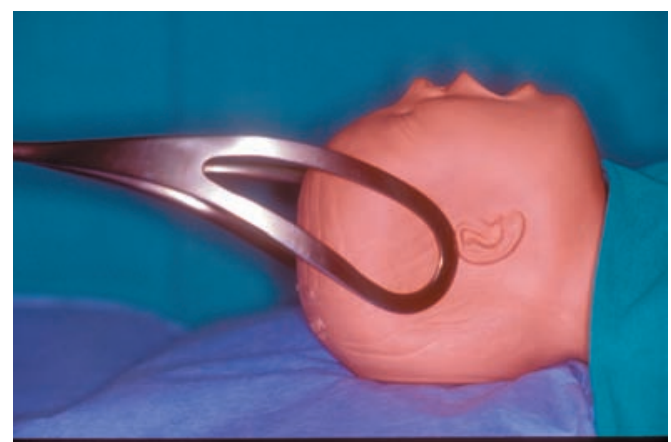

Figura 3. Modo incorrecto de aplicar los Forceps.

fractura deprimida frontal derecha mostrada en la TAC (Fig 2). Fue intervenida de urgencia elevando la depresión. Se remitió a su domicilio tras 5 días postoperatorio; tras dos años de seguimiento es dada de alta definitivamente con TAC normal.

\section{Caso 3}

Niña de 1 día de edad y 40 semanas de gestación que precisó asistencia con forceps en el parto. Sufre una fractura deprimida frontal derecha. Neurológicamente asintomática, fue intervenida realizándose elevación de la fractura. Tras dos años de seguimiento es dada de alta con control por resonancia normal.

\section{Discusión}

El traumatismo durante el parto supone una condición adversa para el feto en su salida por el canal materno ${ }^{10}$. Las lesiones producidas durante el mismo son consideradas graves cuando afectan al plexo braquial o producen fracturas craneales o contusiones cerebrales ${ }^{11}$.

La incidencia real de lesiones del sistema nervioso central es desconocida, pudiendo ser clasificadas en 7 tipos según Hovind ${ }^{6}$ : 1. Hemorragia extracraneal, 2. Fractura
2009; 20: 262-264

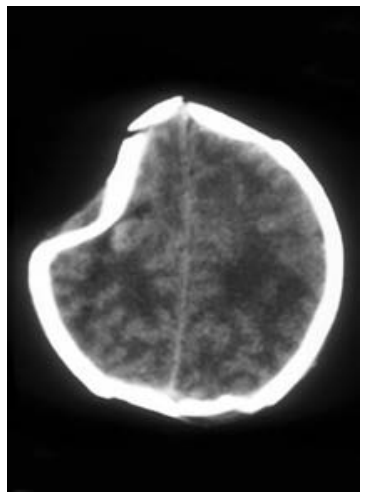

Figura 2. TAC craneal con hundimiento frontal derecho.

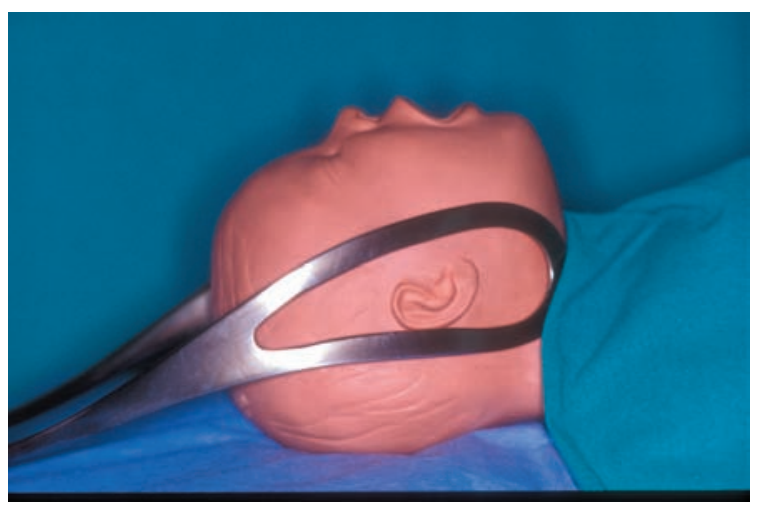

Figura 4. Modo correcto de aplicar los Forceps.

craneal, 3. Hemorragia intracraneal, 4. Contusión cerebral, 5. Contusión cerebelosa, 6. Lesiones espinales y 7. Lesión de nervios periféricos.

La lesión extracraneal hemorrágica más frecuente es el cefalohematoma, que puede ocurrir en un 1 a $2 \%$ de los partos normales. Las fracturas craneales pueden ser lineales, deprimidas o evolutivas. Las lineales suelen asentar en los huesos parietales, con una incidencia de hasta un $10 \%{ }^{4}$. Las fracturas deprimidas suelen ser de poca magnitud, como la fractura en pelota de ping-pong, con una frecuencia de 1 por mil partos.

Muchas de las fracturas en ping-pong se dan en la región parietal, causadas por la aplicación de forceps o por la presión en el propio canal del parto, sobre todo contra el promontorio sacro. Las fracturas deprimidas producidas por traumatismo del parto pueden llegar a suponer el $16 \%$ de las fracturas craneales en estos niños y muchas de ellas son debidas a la aplicación de forceps u otras maniobras agresivas $^{1,3,8.12}$ o por la dificultad de paso por el canal ${ }^{2}$. El $75 \%$ se producen en región parietal y el $15 \%$ son frontales. Las indicaciones de cirugía de reparación son, según Loeser $^{7}$ : a) fragmento óseo libre en parénquima cerebral, b) déficit neurológico, c) aumento de la presión intracraneal y d) fístula de LCR.

Las fracturas evolutivas son un tipo especial de fractura 
el las que siempre hay lesión cerebral y la duramadre está rota. Si es mayor de $4 \mathrm{~mm}$ la pulsación cerebral orada la fractura formándose un pseudomeningocele. El tratamiento es siempre la reparación quirúrgica.

El tercer tipo de lesión es la hemorragia intracraneal, que puede ser epidural, subdural, subaracnoidea, intraventricular o intraparenquimatosa. Los hematomas subdurales pueden producirse por ruptura de venas corticales o lesión de senos venosos, especialmente en las desproporciones pelvicefálicas, prematuros, primípara o multípara añosa con parto prolongado, en presentaciones de frente o nalgas que requieren forceps o ventosa. Clínicamente se observa rigidez nucal, bradicardia, anisocoria, hemiparesia, hipotensión y problemas cardiorrespiratorios. El diagnóstico se realiza mediante TAC o RM. El tratamiento en los casos que lo requieren es la evacuación del hematoma mediante trépanos o craneotomía.

El hematoma epidural supone el 2\% de las hemorragias cerebrales neonatales ${ }^{6}$, la mayoría debidas a ruptura de vasos meníngeos o senos venosos diploicos tras fractura craneal ${ }^{5}$. Estos hematomas epidurales ocurren en partos prolongados y/o en aquéllos asistidos por forceps. Los pacientes muestran fontanela tensa, déficits neurológicos focales, hipotensión... El diagnóstico es realizado de nuevo mediante TAC o RM y el tratamiento consiste igualmente en evacuación con trépanos o pequeña craneotomía.

Las hemorragias subaracnoidea, intraparenquimatosa e intraventricular son raras, siendo más habituales en prematuros de peso inferior a $2 \mathrm{Kg}$ o menores de 30 semanas de gestación, por cambios hipóxicos o isquémicos que pueden añadirse a un parto traumático ${ }^{5}$. Las contusiones cerebrales, cerebelosas o espinales son muy infrecuentes. Sin embargo, las lesiones de nervios periféricos han aumentado su incidencia, con un 5 a 10 por mil nacidos vivos ${ }^{5}$.

\section{Conclusiones}

Las lesiones debidas a un parto traumático han disminuido notablemente en los últimos años dado que la mayoría de partos son atendidos en centros especializados. Además hay un mejor conocimiento de los factores de riesgo, como son las primíparas con fetos de más de $3,5 \mathrm{Kg}$, en las cuales es esencial la correcta aplicación de los forceps (Fig 3 y 4), en las cuales se realizan cesáreas en un $25 \%$ más de casos que hace unos años. La indicación quirúrgica en los recién nacidos depende del balance riesgo-beneficio de la cirugía, siendo clara la indicación en los hundimientos craneales, hematomas intracraneales con déficits neurológicos ${ }^{9}$ o las fracturas evolutivas.

\section{Bibliografía}

1. Alexander, E. Jr., Davis, Ch. Jr.: Intra-uterine fracture of the infant's skull. J. Neurosurg 1969; 30: 446-454.

2. Axton, J.H.M., Levy, L.F.: Congenital moulding depression of the skull. Br J. Med 1965; 1: 1644-1647.

3. Choux, M.: Incidence, diagnosis and management of skull fractures. In: Raimondi AJ, Choux M, Di Rocco C (eds). Head injuries in the newbom and infant. New York Springer Verlag 1986; pp 163-182.

4. Gresham, E.L.: Birth trauma. Pediatr. Clin. North. Am 1975; 22: 317-328.

5. Harpold, T.L., McComb, J.G., Levy, M.L.: Neonatal neurosurgical trauma. Neurosurgery Clinics of North America 1998; 9: 141-154.

6. Hovind, K.H., (1986) Traumatic birth injuries. In: Raimondi AJ, Choux M, Di Rocco C (eds). Head injuries in the newborn and infant. New York, Springer-Verlag pp 87-109.

7. Loeser, J.D., Kilburn, H.L., Jollet, T.: Management of depressed skull fracture in the newborn. J. Neurosurg. 1976; 44: 62-64.

8. Papaefthymiou, G., Oberbauer, R., Pendl, G.: Craniocerebral birth traume caused by vacuum extraction: a cause of growing skull fracture as a perinatal complication. Child's Nery Syst 1996; 12:117-120.

9. Poch, J.M., Nogues, P., Cabañero, M., Boix, J.: Un recién nacido con traumatismo obstétrico. An. Esp. Pediatr. 1997; 47: 435-436.

10. Potter, E.L.: Pathology of the fetus and infant. $2^{\text {nd }}$ ed. Chicago, Year Book 1961.

11. Puza, S., Roth, N., Macones, G.A., Mennuti, M.T., Morgan, M.A.: Does cesarean section decreese the incidence of major birth trauma? J. Perinatol 1998, 18: 9-1.

12. Till, K.: Pediatric neurosurgery. Oxford, Blackweil Scientific 1975, pp 109-113

Villarejo, F.; Belinchón, J.M.; Carceller, F.; Gómez-Sierra, A.; Pascual, A.; Cordobés, F.; Pérez Díaz, C.; Rivero, B.: Lesiones craneales secundarias a parto asistido con forceps. Neurocirugía 2009; 20: 262-264.

Correspondencia postal: José Maria Belinchón de Diego. Servicio de Neurocirugía. Hospital General Universitario de Valencia. Av Tres Cruces s/n. 46014 Valencia. 